\title{
Development and application of a multiplex PCR method \\ for the simultaneous detection and differentiation of feline panleukopenia virus, feline bocavirus, and feline astrovirus
}

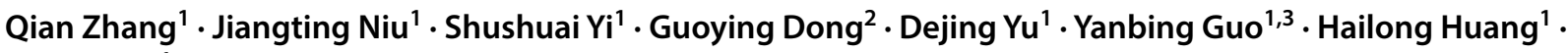 \\ Guixue $\mathrm{Hu}^{1}$
}

Received: 21 March 2019 / Accepted: 5 August 2019 / Published online: 10 September 2019

(c) Springer-Verlag GmbH Austria, part of Springer Nature 2019

\begin{abstract}
A multiplex polymerase chain reaction (mPCR) assay was developed to detect and distinguish feline panleukopenia virus (FPV), feline bocavirus (FBoV) and feline astrovirus (FeAstV). Three pairs of primers were designed based on conserved regions in the genomic sequences of the three viruses and were used to specifically amplify targeted fragments of $237 \mathrm{bp}$ from the VP2 gene of FPV, $465 \mathrm{bp}$ from the NP1 gene of FBoV and $645 \mathrm{bp}$ from the RdRp gene of FeAstV. The results showed that this mPCR assay was effective, because it could detect at least 2.25-4.04 $\times 10^{4}$ copies of genomic DNA of the three viruses per $\mu \mathrm{l}$, was highly specific, and had a good broad-spectrum ability to detect different genotypes of the targeted viruses. A total of 197 faecal samples that had been screened previously for FeAstV and FBoV were collected from domestic cats in northeast China and were tested for the three viruses using the newly developed mPCR assay. The total positive rate for these three viruses was 59.89\% (118/197). From these samples, DNA from FPV, FBoV and FeAstV was detected in 73, 51 and 46 faecal samples, respectively. The mPCR testing results agreed with the routine PCR results with a coincidence rate of $100 \%$. The results of this study show that this mPCR assay can simultaneously detect and differentiate FPV, FBoV and FeAstV and can be used as an easy, specific and efficient detection tool for clinical diagnosis and epidemiological investigation of these three viruses.
\end{abstract}

\section{Introduction}

Cats are among the most common companion animals and play an important role in providing emotional support for people. To date, the population of domestic cats kept by

Handling Editor: Sheela Ramamoorthy.

Qian Zhang and Jiangting Niu contributed equally to this work.

Electronic supplementary material The online version of this article (https://doi.org/10.1007/s00705-019-04394-8) contains supplementary material, which is available to authorized users.

Guixue $\mathrm{Hu}$

huguixue901103@126.com

1 College of Animal Science and Technology, Jilin Agricultural University, Changchun 130118, Jilin, China

2 College of Global Change and Earth System Science, Beijing Normal University, Beijing 100875, China

3 Jilin Institute of Animal Husbandry and Veterinary Science, Changchun 130062, Jilin, China humans worldwide is estimated at 80-400 million [17], and humans are increasingly paying more attention to the health of cats. Viral diarrhea is common in cats, especially in young kittens, and it is also a major threat to their health. It is commonly recognized that feline panleukopenia virus (FPV) is the major virus that causes diarrhea in cats [21]. Over the past few years, more diarrhea-related viruses, such as feline bocavirus (FBoV) [10], feline astrovirus (FeAstV) [11] and feline kobuvirus (FeKoV) [4], have been described in cats, and new detection techniques have been developed and applied [17].

FPV, a member of the species Carnivore protoparvovirus 1, which belongs to the genus Protoparvovirus within the family Parvoviridae, is a highly contagious pathogen in domestic and wild cats [1]. FPV is transmitted by the faecal-oral route and primarily infects young kittens aged 3-6 months [15, 21], resulting in severe enteric and immunosuppressive diseases characterized by fever, depression, anorexia, vomiting, acute diarrhea, haemorrhagic enteritis and leukopenia [16]. At present, FPV is widely spread throughout the world, with a high mortality rate of $25 \%$ to $90 \%$ [18], 
and represents a serious threat to the life and health of cats. Furthermore, infections with canine parvovirus $2 \mathrm{a}, 2 \mathrm{~b}$ and 2c (CPV-2a, -2b and -2c) have also been described in domestic cats in many countries [18]. CPV-2 and FPV belong to the same viral species, Carnivore protoparvovirus 1, within the family Parvoviridae [6]. FeAstV, species Mamastrovirus 2, belongs to the genus Mamastrovirus within the family Astroviridae and is a non-enveloped, single-stranded positive RNA virus [11]. FeAstV was first reported in 1981 in faeces from diarrheic cats [9], and a novel FeAstV genotype was found in 2012 [11]; however, studies on FeAstV pathogenicity are limited. FBoV was first detected in a stray cat in Hong Kong in 2012 [10] and has been reported in the USA [27], Portugal [17], Japan [22] and China [25]. According to the latest report of the International Committee on Taxonomy of Viruses (ICTV), FBoV-1, FBoV-2 and FBoV-3 have been recognized as members of the species Carnivore bocaparvovirus 3 , 4, and 5, respectively, within the genus Bocaparvovirus [5], based on the amino acid sequence of the complete NS1 gene [25]. Epidemiological investigations in different countries have suggested that FeAstV and FBoV may cause diarrhea in cats with a prevalence of $4.8 \%-23.4 \%$ $[3,14,20,24]$ and $5.5 \%-25.9 \%[10,22,25,27]$, respectively, and coinfections with FPV, FBoV and FeAstV are also common in cats with diarrhea. Because of the similar clinical symptoms and frequency of coinfections with these three viruses, differentiation of these viruses during clinical diagnosis and epidemiological investigations is difficult.

PCR has become the most widely used method for detection of pathogens due to its high sensitivity and specificity. Routine PCR/RT-PCR methods for detection of FPV, FeAstV and FBoV have been described in previous reports $[12,16]$, but ??there have been no reports of?? the application of routine PCR for the simultaneous detection and differentiation of these three viruses. Multiplex PCR (mPCR) can simultaneously detect and distinguish two or more target viruses in one reaction, can save time and cost, and has been widely used in large-scale epidemiological investigations [8, 28]. In the present study, a mPCR assay was developed by combining three pairs of universal primers targeting different members of the species Carnivore protoparvovirus 1, FBoV and FeAstV in one reaction. This mPCR assay can be used as an easy and effective method for the detection and differentiation of these three viruses in clinical samples.

\section{Materials and methods}

\section{Viruses}

The following viruses were used as positive virus controls in the present study: FPV strain JL-03/17-05 (GenBank accession number MF541125), FBoV strain 17CC0302
(GenBank accession number MH155947), and FeAstV strain 17CC0502 (GenBank accession number MH253845). Moreover, feline kobuvirus (FeKoV) strain 16CC0802 (GenBank accession number MH159805), feline calicivirus (FCV) strain CH-JL2 (GenBank accession number KJ495725), feline herpesvirus type 1 (FHV-1) strain CH-B, and standard positive DNA controls of feline coronavirus (FCoV), feline leukemia virus (FeLV) and feline immunodeficiency virus (FIV) were used to test the specificity of the mPCR. Different strains identified and maintained in our laboratory, including FPV, CPV 2, CPV 2a, CPV 2b, FBoV 1, FBoV 2, FBoV 3, FeAstV-1 and FeAstV-2, were used to test the ability of the mPCR method to detect a broad spectrum of viruses.

\section{Nucleic acid extraction}

Viral nucleic acids were extracted from positive and negative virus controls using an AxyPrep ${ }^{\mathrm{TM}}$ Body Viral DNA/RNA Miniprep Kit (Axygen, China) according to the manufacturer's instructions. Reverse transcription was performed for RNA viruses, including FeAstV, FeKoV and FCV, for cDNA synthesis using a PrimerScript ${ }^{\mathrm{TM}}$ RT Reagent Kit (Takara, China) according to the manufacturer's instructions. Nucleic acids extracted from DNA viruses were used directly as a template for PCR. The clinical samples were prepared using a previously described protocol [26], and nucleic acids were extracted using the above methods.

\section{Primer design}

The reference genomic sequences of FPV (FPV, CPV-2, CPV-2a, CPV-2b and CPV-2c), FBoV (FBoV 1, FBoV 2 and FBoV 3) and FeAstV (FeAstV-1 and FeAstV-2) were obtained from the GenBank nucleotide sequence database from the National Center for Biotechnology Information (NCBI). Highly conserved regions of the same virus were discovered by multiple alignment using the MEGA 7.0 software. Then, three pairs of specific primers based on the conserved regions of these viruses, the VP2 gene of FPV, the NP1 gene of FBoV, and the RdRp gene of FeAstV, were designed for the mPCR assay. Primer Blast and MFE primer software were used to determine the $T_{m}$ value and specificity of these primer pairs. The nucleotide sequences of the primers are provided in Table 1.

\section{Standard plasmid template preparation}

Routine PCR was performed for each virus with the corresponding primers in a $25-\mu 1$ reaction mixture including 12.5 $\mu \mathrm{l}$ of Premix Ex Taq, $0.5 \mu \mathrm{l}$ each of the forward and reverse primers $(10 \mu \mathrm{M}), 2 \mu \mathrm{l}$ of the nucleic acid extracted from the targeted virus, and $9.5 \mu 1$ of RNase-free water. The reactions 
Table 1 Nucleotide sequences of the primers used for the multiplex PCR assay

\begin{tabular}{lllll}
\hline Target virus & Target gene & Primer name & Nucleotide sequence (5'-3') & $\begin{array}{l}\text { Ampli- } \\
\text { con size } \\
\text { (bp) }\end{array}$ \\
\hline FPV & VP2 & FPV-F & CATACATGGCAAACAAATAGAGCA & 237 \\
& & FPV-R & TGTTTTAAATGGCCCTTGTGTAGA & \\
FBoV & NP1 & FBoV-F & AGAACCRCCRATCACARTCCACT & 465 \\
& & FBoV-R & TGGCRACCGCYAGCATTTCA & \\
FeAstV & RdRp & FeAstV-F & GCGGATTGGGCATGGTTTAGA & 645 \\
& & FeAstV-R & ACCCCTCGTTTGGATCGTTACCT & \\
\hline
\end{tabular}

were performed under the following conditions: initial denaturation at $94{ }^{\circ} \mathrm{C}$ for $5 \mathrm{~min}$, followed by 35 cycles of $94{ }^{\circ} \mathrm{C}$ for $45 \mathrm{~s}, 51^{\circ} \mathrm{C}$ for $45 \mathrm{~s}$ and $72{ }^{\circ} \mathrm{C}$ for $30 \mathrm{~s}$ and a final extension at $72{ }^{\circ} \mathrm{C}$ for $10 \mathrm{~min}$. The different-sized products of the three viruses, 237 bp for FPV, 465 bp for FBoV and 645 bp for FeAstV, were purified using an AxyPrep ${ }^{\mathrm{TM}}$ DNA Gel Extraction Kit (Axygen, China) and ligated to the pMD$18 \mathrm{~T}$ vector (Takara, China) according to the manufacturer's instructions. The recombinant plasmids identified as positive were named pMD-FPV, pMD-FBoV and pMD-FeAstV, respectively, and were used to optimize the reaction conditions and determine the sensitivity of the mPCR assay. The plasmid copy number was calculated according to the following formula: copy number $($ copies $/ \mu \mathrm{l})=\left[6.02 \times 10^{23} \times\right.$ plasmid concentration $(\mathrm{ng} / \mu \mathrm{l})] /[$ plasmid length $(\mathrm{bp}) \times 660]$.

\section{Optimization of mPCR}

The plasmid DNA of the three viruses was serially tenfold diluted to concentrations of $1 \times 10^{6}$ copies $/ \mu 1,1 \times 10^{5}$ copies/ $\mu 1$ and $1 \times 10^{4}$ copies/ $\mu 1$. Diluted mixtures containing pMD-FPV, pMD-FBoV and pMD-FeAstV at equal concentrations and volumes were used as templates to optimize the reaction mixture and conditions of the $\mathrm{MPCR}$ assay, including the concentrations of the primers, dNTP and Ex Taq enzyme; the annealing temperature; and the number of cycles of PCR. Specifically, mPCR was performed in a total volume of $25 \mu \mathrm{l}$ of reaction mixture containing $2 \mu \mathrm{l}$ of each plasmid DNA, $2.5 \mu$ l of 10× PCR buffer, $0.25-2 \mu$ of each primer at a concentration of $10 \mu \mathrm{M}, 1-4 \mu \mathrm{l}$ of the dNTP mix (2.5 mM), 0.3-1 $\mu$ l of Ex Taq enzyme (5 U/ $\mu \mathrm{l})$ (Takara, China), and RNase-free water to a total volume of $25 \mu \mathrm{l}$. The reactions were performed under the following conditions in a thermal cycler: predenaturation at $94{ }^{\circ} \mathrm{C}$ for $5 \mathrm{~min}$, followed by $30-40$ cycles of $94{ }^{\circ} \mathrm{C}$ for $45 \mathrm{~s}, 50-62{ }^{\circ} \mathrm{C}$ for $45 \mathrm{~s}$, and $72{ }^{\circ} \mathrm{C}$ for $30 \mathrm{~s}$ and a final extension at $72{ }^{\circ} \mathrm{C}$ for $10 \mathrm{~min}$. A 5 - $\mu$ l sample of the PCR reaction mixture from each test was subjected to electrophoresis on a $2 \%$ agarose gel and visualized using a gel documentation system (Wealtec, USA). The optimal reaction conditions were determined based on the presence or absence of primer dimers and the grey-scale value of the electrophoresis band evaluated using Image $\mathbf{J}$ software.

\section{Specificity of the MPCR assay}

To evaluate the specificity of the MPCR, various combinations of positive virus controls, including a single plasmid DNA, a mixture of two plasmids, and a mixture of three plasmids, were used as templates in the different reactions. The different-sized PCR products were purified and sequenced to confirm the specificity of the mPCR. mPCR was also used to detect other non-targeted viruses, including FeKoV, FCV, FHV-1, FCoV, FIV and FeLV. RNase-free water was used as a negative control.

\section{Sensitivity of the MPCR assay}

Tenfold serially diluted recombinant plasmids containing sequences from the three viruses, ranging from $4.04 \times 10^{9}$ copies/ $\mu$ l to $4.04 \times 10^{0}$ copies/ $\mu$ l for FPV, $3.13 \times 10^{9}$ copies/ $\mu \mathrm{l}$ to $3.13 \times 10^{0}$ copies $/ \mu \mathrm{l}$ for $\mathrm{FBoV}$, and $2.25 \times 10^{9}$ copies $/ \mu \mathrm{l}$ to $2.25 \times 10^{0}$ copies $/ \mu$ l for FeAstV, were used to determine the sensitivity of the mPCR. The sensitivity of the routine PCR with only one pair of primers was also tested using tenfold serial dilutions.

\section{Ability of the MPCR assay to detect multiple genotypes}

Different genotypes of the three viruses, including FPV, CPV-2, CPV-2a, CPV-2b, FBoV-1, FBoV-2, FBoV-3, FeAstV-1 and FeAstV-2, were tested using the mPCR assay to determine the breadth of the specificity of this method.

\section{Application of the MPCR assay on clinical samples}

A total of 197 faecal samples collected from domestic cats in northeast China were used to evaluate the performance of mPCR using clinical samples. These clinical samples had been used to investigate the prevalence of FBoV and FeAstV using conventional single PCR in a previous study 
performed in our laboratory $[24,25]$. In the present study, these clinical samples were used to detect FPV using routine PCR as reported previously [23]. The positive rate was calculated for each virus, and the difference in the prevalence of each virus between diarrheic and healthy cats was evaluated by the two-tail chi square test using PASW Statistic 19.0. A probability $(p)$ value $<0.05$ was considered statistically significant.

\section{Results}

\section{Establishment of the MPCR assay}

After optimization, the primers for FPV, FBoV and FeAstV produced amplicons of $237 \mathrm{bp}, 465 \mathrm{bp}$ and $645 \mathrm{bp}$, respectively, with clear target bands on an agarose gel. According to the evaluation method described above, the optimization results were in agreement using different concentrations of the DNA plasmid mixture as templates. The optimal annealing temperature was $51{ }^{\circ} \mathrm{C}$, and the optimal cycle number was 40 (Supplementary Fig. 1). The concentrations of the various reagents in the reaction mixture were also optimized in this study (Supplementary Fig. 2), and the final concentrations were as follows: $2 \mu \mathrm{l}$ of the $\mathrm{dNTP} \operatorname{mix}(2.5 \mathrm{mM}), 0.5 \mu \mathrm{l}$ of Ex Taq DNA polymerase ( $5 \mathrm{U} / \mu \mathrm{l})$, and $0.5 \mu \mathrm{l}$ each of the forward and reverse primers $(10 \mu \mathrm{M})$ for the three viruses.

\section{MPCR specificity}

The specificity testing results showed that amplicons of the expected sizes were produced targeting the corresponding virus when different combinations of FPV, FBoV and FeAstV were used as templates in mPCR (Fig. 1). The identity of these amplicons was confirmed by sequencing. No amplification occurred when FeKoV, FCV, FHV-1, FCoV, FIV, FeLV or RNase-free water were used as template in the mPCR. These results indicate that the newly developed
mPCR assay was highly specific for the detection and differentiation of FPV, FBoV and FeAstV.

\section{mPCR sensitivity}

To determine the sensitivity of the mPCR, plasmid DNA containing sequences from the three viruses was serially diluted tenfold and used as a template for $\mathrm{mPCR}$. The minimum detection limit of mPCR was $4.04 \times 10^{3}, 3.13 \times 10^{3}$ and $2.25 \times 10^{4}$ viral copies for FPV, FBoV and FeAstV, respectively (Fig. 2d). Moreover, the sensitivity testing results of routine PCR with only one pair of primers showed that the minimum detection limit for FPV, FBoV and FeAstV was $4.04 \times 10^{2}, 3.13 \times 10^{2}$ and $2.25 \times 10^{3}$ viral copies, respectively (Fig. 2a-c). Taken together, the sensitivity results show that the sensitivity of the mPCR was slightly higher than that of routine PCR.

\section{Ability of the MPCR assay to detect multiple genotypes}

mPCR was used to detect different genotypes of the three viruses to determine the breadth of the specificity of this method. The results showed that the MPCR was able to detect different genotypes of the target viruses, including FPV, CPV-2, CPV-2a, CPV-2b, FBoV 1, FBoV 2, FBoV 3 , FeAstV-1 and FeAstV-2, and amplicons of the expected sizes were obtained (Supplementary Fig. 3).

\section{Evaluation of the MPCR assay using clinical samples}

A total of 197 faecal samples that had previously been screened for $\mathrm{FBoV}$ and $\mathrm{FeAstV}$ were tested using the newly developed mPCR assay and routine PCR targeting FPV, and the test results for $\mathrm{mPCR}$ were then compared to those of routine PCR. The results presented in Table 2 indicate that the total detection rate for these three viruses was 59.89\% (118/197), of which 73 samples were positive for FPV, 51 were positive for FBoV,

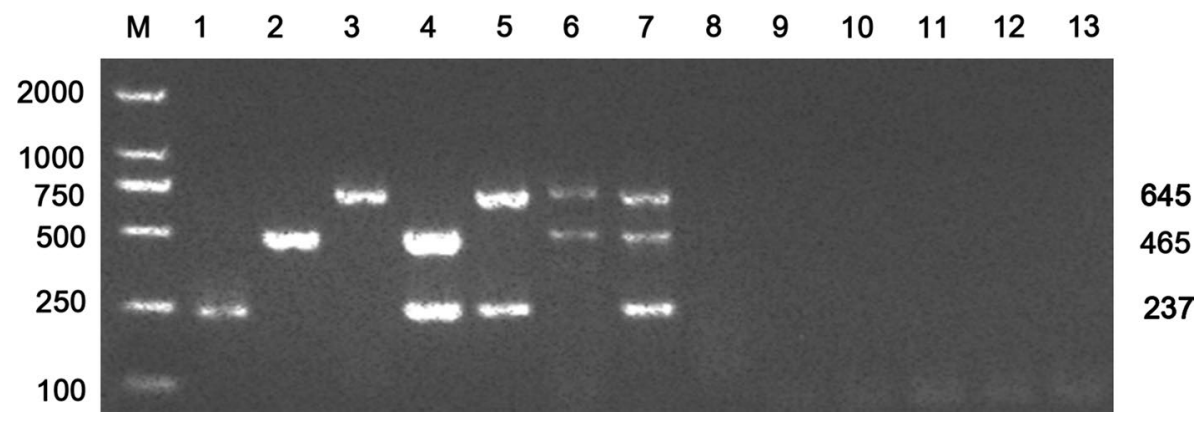

Fig. 1 Specificity of multiplex PCR for the detection of positive and negative controls using mixed primers. Lanes 1-13: 1, FPV; 2, FBoV; 3, FeAstV; 4, FPV and FBoV; 5, FPV and FeAstV; 6, FBoV and
FeAstV; 7, FeAstV, FBoV and FPV; 8, FCV; 9: FHV-1; 10, FCoV; 11, FeLV; 12, FIV; 13, negative control; M, DL2000 DNA marker 

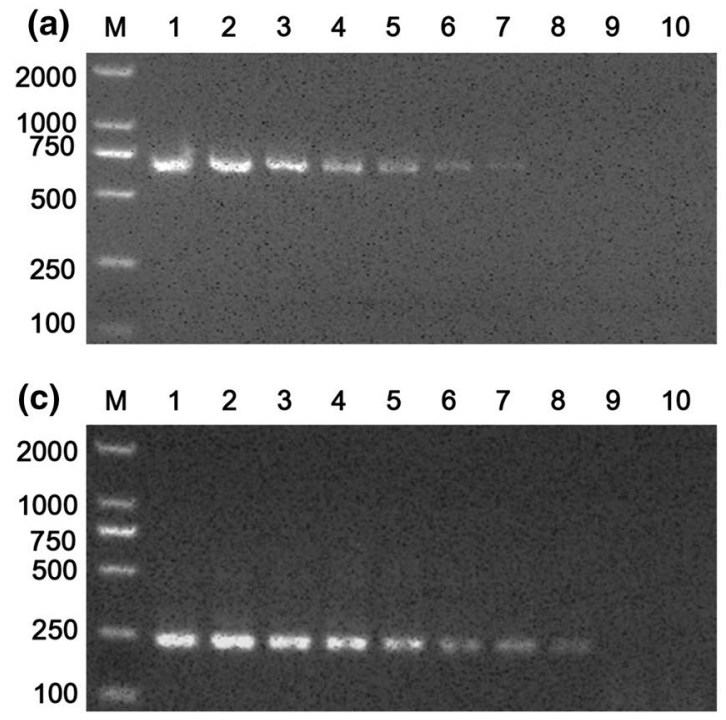

Fig. 2 Sensitivity of multiplex PCR and routine PCR for the detection of tenfold serially diluted plasmid DNA from FPV, FBoV and FeAstV. (a, b, c) Routine PCR for FeAstV, FBoV and FPV; (d) mPCR. Lanes 1-10 ( $a, b$ and c), FeAstV-, FBoV- and FPV-positive plasmid concentrations ranging from $2.25 \times 10^{9}$ to $2.25 \times 10^{0}$ copies/ $\mu 1,3.13 \times 10^{9}$ to $3.13 \times 10^{0} \mathrm{copies} / \mu \mathrm{l}$ and $4.04 \times 10^{9}$ to $4.04 \times$

and 46 were positive for $\mathrm{FeAst} \mathrm{V}$ according to the $\mathrm{mPCR}$ assay, which was in $100 \%$ agreement with the results of the routine PCR assays. The total detection rate of the three viruses in cats with diarrhea $(73.33 \%, 77 / 105)$ was significantly higher than that in healthy cats $(44.57 \%, 41 / 92)$. Moreover, the detection results showed that the total positive rate for FPV $(37.06 \%$, 73/197) was the highest, followed by FBoV $(25.89 \%, 51 / 197)$ and FeAstV (23.35\%, 46/197), and that the positive rate for each virus in domestic cats with diarrhea was significantly higher than that in healthy cats $(47.62 \%$ versus $25 \%$ for FPV, $33.33 \%$ versus $17.39 \%$ for $\mathrm{FBoV}$ and $36.19 \%$ versus $8.69 \%$ for FeAstV) (Fig. 3a). Coinfections were frequent, with 34.75\% (41/118) of cats shedding more than one of the three viruses.

We also analysed the differences in the monoinfection and coinfection rates for each virus between diarrheic and healthy cats (Fig. 3b). The monoinfection rates for FPV (86.95\%, 20/23), FBoV $(62.5 \%, 10 / 16)$ and FeAstV $(62.5 \%, 5 / 8)$ were higher than the coinfection rates in healthy cats. In contrast, cats with diarrhea had higher coinfection rates, with values of $62 \%$ (31/50) for FPV, $51.43 \%$ (18/35) for FBoV, and 84.22\% (32/38) for FeAstV, respectively. Moreover, the coinfection rates of the three viruses in diarrheic cats were significantly higher than those in healthy cats.
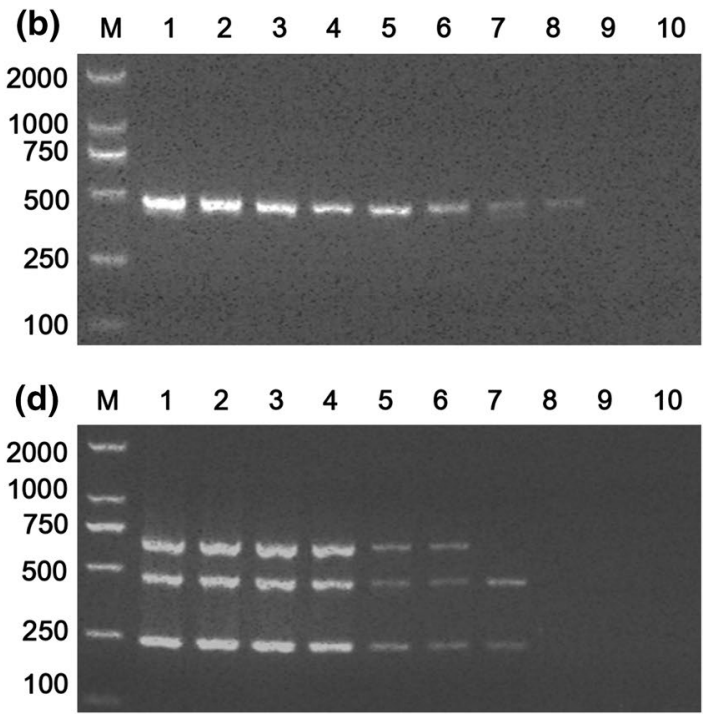

$10^{0}$ copies/ $\mu$ l. Lanes 1-10 in (d) are reactions performed with tenfold serial dilutions of mixtures of plasmids containing sequences from the three viruses $\left(2.25 \times 10^{9}-2.25 \times 10^{0}\right.$ copies $/ \mu 1,3.13 \times 10^{9}-3.13$ $\times 10^{\circ}$ copies $/ \mu \mathrm{l}$, and $4.04 \times 10^{9}-4.04 \times 10^{0}$ copies $/ \mu 1$ of each virus/ sample). M, DL2000 DNA marker

\section{Discussion}

Viral diarrhea is the most common clinical disease in domestic cats and seriously threatens the life and health of cats. FPV is generally considered in the clinical diagnosis of viral diarrhea in cats, while other viruses are often ignored in clinical testing. Recently, some novel enteric viruses, including $\mathrm{FBoV}, \mathrm{FeAstV}$ and $\mathrm{FeKoV}$ [13], were found in domestic cats, and studies suggest that these viruses are associate with viral diarrhea in cats. Over the last two years, our laboratory has investigated the prevalence of new feline enteric viruses in northeast China and has found that FBoV and $\mathrm{FeAstV}$ are widespread in China, with a high prevalence of approximately $25 \%[24,25]$. Furthermore, coinfections with $\mathrm{FPV}, \mathrm{FBoV}$ and FeAstV were also found to be frequent in our investigation. However, there is no scientific detection assay to test for the three viruses. Therefore, a multiplex PCR assay that can simultaneously detect and differentiate FPV, FBoV and FeAstV in one reaction was developed and evaluated in the present study.

The competition between two or more pairs of primers in multiplex PCR may affect its specificity and sensitivity, 


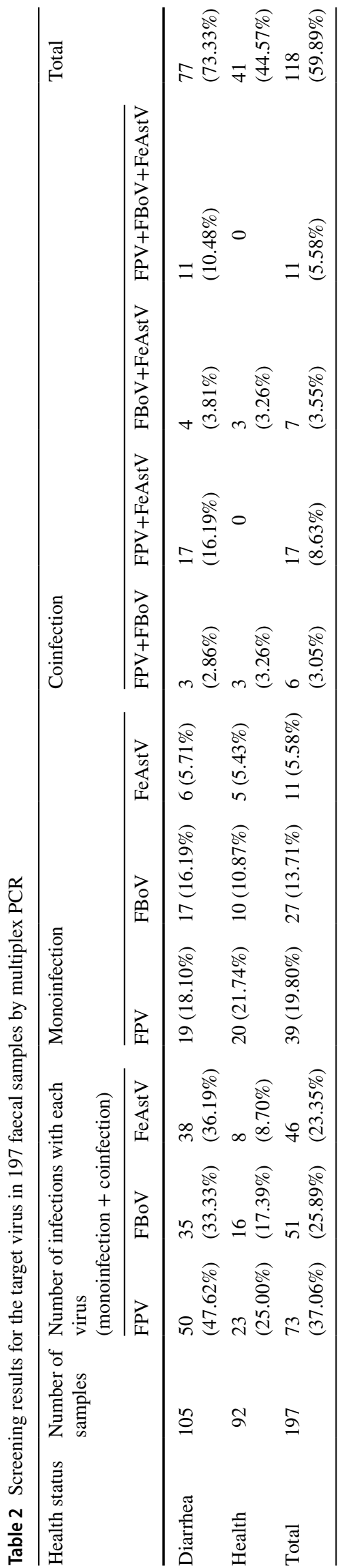

so primer design and selection are critical for the development of multiplex PCR assays. In this study, three pairs of primers targeting highly conserved regions of the genomic sequences of FPV, FBoV and FeAstV were designed to detect different genotypes of the same virus. The primers' annealing temperature and amplicon length were also evaluated using the online software MFE primer. The primer combination produced amplicons of $237 \mathrm{bp}$ for FPV, 465 bp for FBoV and 645 bp for FeAstV, which were easy to distinguish from one another by electrophoresis using $2.0 \%$ agarose gels. Then, a multiple PCR method with this primer combination was established by optimizing the reagent concentration and reaction conditions.

Various common viruses in cats, including FeKoV, FCV, FHV-1, FCoV, FIV and FeLV, were used to evaluate the specificity of the mPCR assay. The testing results showed that no cross-reactions or nonspecific reactions were produced in mPCR with these viruses as templates (Fig. 1), suggesting that this assay had good specificity. Sensitivity testing showed that the minimum detection limit of mPCR was $4.04 \times 10^{3}$ copies $/ \mu 1,3.13 \times 10^{3}$ copies $/ \mu 1$ and $2.25 \times$ $10^{4}$ copies/ $\mu \mathrm{l}$ for FPV, FBoV and FeAstV, respectively, and this was 10-100-fold lower than that of routine PCR (Fig. 2). The low sensitivity was caused by the interaction of primers, as described in previous studies [7, 26]. However, in the detection of clinical samples, the mPCR and routine PCR results were in $100 \%$ agreement, suggesting that $\mathrm{mPCR}$ was effective.

It has been demonstrated that not only FPV can infect cats but also that the different subtypes of CPV-2 (CPV2a, CPV-2b and CPV-2c) can infect domestic cats, causing diarrhea [2]. In addition, in previous studies, FBoV has been classified into three genotypes (FBoV-1, FBoV-2 and FBoV-3) [22] and FeAstV has been classified into different genotypes [24]. In the present study, we designed three pairs of universal primers that can detect the different genotypes of the three viruses. The mPCR was able to detect nucleic acids extracted from different genotypes of the target virus (Supplementary Fig. 3), suggesting that this method detects a broad spectrum of variants.

Out of the 197 faecal samples tested, 118 were positive for one or more viruses, with a high positive rate of $59.89 \%$. The total positive rates of FPV, FBoV and FeAstV were $37.06 \%$ (73/197), $25.89 \%$ (51/197) and $23.35 \%$ (46/197), respectively, and the positive rates for all three viruses in cats with diarrhea were higher than those in healthy cats, suggesting that FPV, FBoV and FeAstV are associated with viral diarrhea in cats. Moreover, healthy cats had higher monoinfection rates, while cats with diarrhea had higher coinfection rates. Furthermore, coinfections with two or three viruses were frequent, especially in cats with diarrhea $[17,19]$, with a high positive rate of $34.75 \%$ (41/118), consistent with a previous report [24]. 


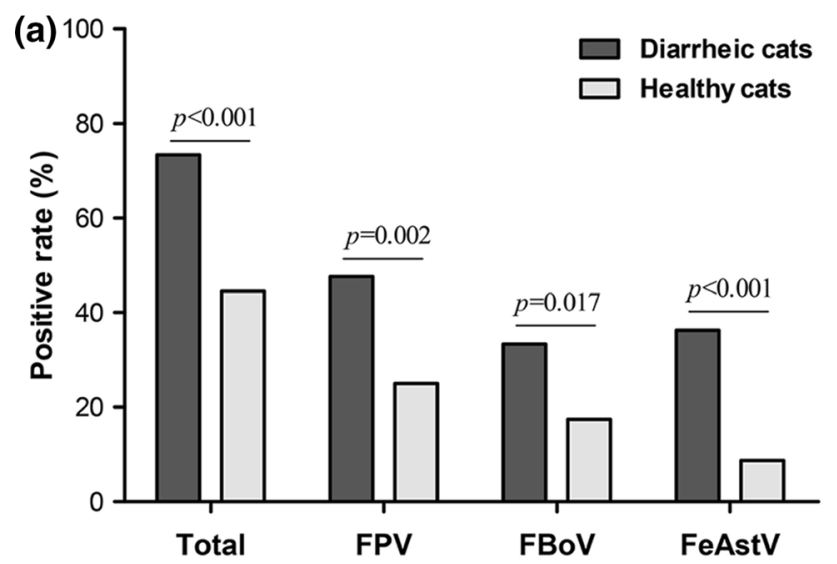

Fig. 3 Prevalence of FPV, FBoV and FeAstV in diarrheic and healthy cats. (a) Total positive rates of these three viruses in diarrheic and healthy cats. The difference was evaluated using the chi-square test.

The data obtained from the present study confirm that coinfections with various enteric viruses are common in domestic cats.

The lack of identification of other feline diarrhea-associated viruses using the mPCR assay is a limitation of this study. First, in this study, we also tried to develop mPCR for FPV, FBoV, FeAstV, feline coronavirus and feline kobuvirus, but the results were not good. We think that primer competition affects the specificity and sensitivity of mPCR. Second, the epidemiological investigations of feline diarrhea-associated viruses performed in our laboratory suggest that the prevalence of FPV, FBoV and FeAst $\mathrm{V}$ is higher in healthy and diarrheic cats and that mixed infections with these three viruses are common in clinical cases. The establishment of this method provides a convenient and advantageous test for epidemiological investigations of these three viruses. However, there are fewer types of viruses involved in epidemiological investigations of feline diarrhea-associated viruses, which is a limitation of this paper. In future studies, we will try to establish an mPCR assay for additional feline diarrheaassociated viruses.

In conclusion, we have developed a multiplex PCR assay that can simultaneously detect and differentiate FPV, FBoV and FeAstV. This multiplex PCR assay is easy, specific, and efficient, and can detect a broad spectrum of different genotypes of these three viruses, thereby providing a good tool for clinical diagnosis or extensive epidemiological investigations of FPV, FBoV and FeAstV.

Funding This study was supported by the Research Project of the National Key Research and Development Plan of China (Grant no. 2016YFD0501002). This work was also supported by the National Key R\&D Program of China (Grant no. 2017YFD0501703).

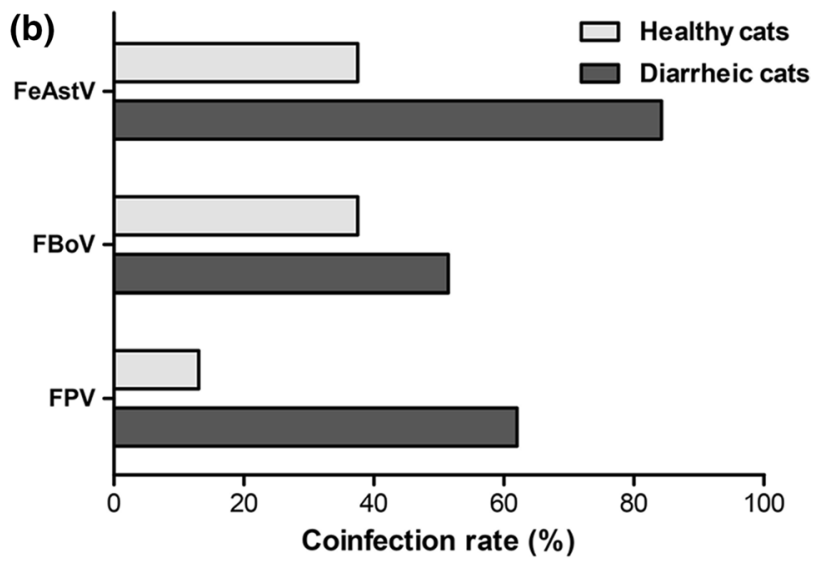

Probability $(p)$ values are shown on the bar chart. A $p$-value $<0.05$ was considered statistically significant. (b) Monoinfection and coinfection rates of these three viruses in diarrheic and healthy cats

\section{Compliance with ethical standards}

Conflict of interest All the authors declare that they have no conflict of interest.

Ethical approval All animal experiments conformed to the guidelines and regulatory requirements established by the Animal Care and Use Committee of Jilin Agricultural University, Jilin Province, China. Owners' consent was obtained to collect samples from healthy and diarrheic cats.

\section{References}

1. Balboni A, Bassi F, De Arcangeli S, Zobba R, Dedola C, Alberti A, Battilani M (2018) Molecular analysis of carnivore Protoparvovirus detected in white blood cells of naturally infected cats. BMC Vet Res 14:41

2. Battilani M, Balboni A, Ustulin M, Giunti M, Scagliarini A, Prosperi S (2011) Genetic complexity and multiple infections with more Parvovirus species in naturally infected cats. Vet Res 42:43

3. Cho YY, Lim SI, Kim YK, Song JY, Lee JB, An DJ (2014) Molecular characterisation and phylogenetic analysis of feline astrovirus in Korean cats. J Feline Med Surg 16:679-683

4. Chung JY, Kim SH, Kim YH, Lee MH, Lee KK, Oem JK (2013) Detection and genetic characterization of feline kobuviruses. Virus Genes 47:559-562

5. Cotmore SF, Agbandje-McKenna M, Chiorini JA, Mukha DV, Pintel DJ, Qiu J, Soderlund-Venermo M, Tattersall P, Tijssen P, Gatherer D, Davison AJ (2014) The family Parvoviridae. Arch Virol 159:1239-1247

6. Decaro N, Buonavoglia C (2012) Canine parvovirus-a review of epidemiological and diagnostic aspects, with emphasis on type 2c. Vet Microbiol 155:1-12

7. Deng X, Zhang J, Su J, Liu H, Cong Y, Zhang L, Zhang K, Shi N, Lu R, Yan X (2018) A multiplex PCR method for the simultaneous detection of three viruses associated with canine viral enteric infections. Arch Virol 163:2133-2138

8. He YP, Zhang Q, Fu MZ, Xu XG (2017) Development of multiplex PCR for simultaneous detection and differentiation of six 
DNA and RNA viruses from clinical samples of sheep and goats. J Virol Methods 243:44-49

9. Hoshino Y, Zimmer JF, Moise NS, Scott FW (1981) Detection of astroviruses in feces of a cat with diarrhea. Brief report. Arch Virol 70:373-376

10. Lau SK, Woo PC, Yeung HC, Teng JL, Wu Y, Bai R, Fan RY, Chan KH, Yuen KY (2012) Identification and characterization of bocaviruses in cats and dogs reveals a novel feline bocavirus and a novel genetic group of canine bocavirus. J Gen Virol 93:1573-1582

11. Lau SK, Woo PC, Yip CC, Bai R, Wu Y, Tse H, Yuen KY (2013) Complete genome sequence of a novel feline astrovirus from a domestic cat in Hong Kong. Genome Announc 1:e00708-13

12. Liu C, Liu F, Li Z, Qu L, Liu D (2018) First report of feline bocavirus associated with severe enteritis of cat in Northeast China, 2015. J Vet Med Sci 80:731-735

13. Lu G, Zhang X, Luo J, Sun Y, Xu H, Huang J, Ou J, Li S (2018) First report and genetic characterization of feline kobuvirus in diarrhoeic cats in China. Transbound Emerg Dis 65:1357-1363

14. Marshall JA, Kennett ML, Rodger SM, Studdert MJ, Thompson WL, Gust ID (1987) Virus and virus-like particles in the faeces of cats with and without diarrhoea. Aust Vet J 64:100-105

15. Mende K, Stuetzer B, Sauter-Louis C, Homeier T, Truyen U, Hartmann K (2014) Prevalence of antibodies against feline panleukopenia virus in client-owned cats in Southern Germany. Vet J 199:419-423

16. Moschidou P, Martella V, Lorusso E, Desario C, Pinto P, Losurdo M, Catella C, Parisi A, Banyai K, Buonavoglia C (2011) Mixed infection by Feline astrovirus and Feline panleukopenia virus in a domestic cat with gastroenteritis and panleukopenia. J Vet Diagn Investig 23:581-584

17. Ng TF, Mesquita JR, Nascimento MS, Kondov NO, Wong W, Reuter G, Knowles NJ, Vega E, Esona MD, Deng X, Vinje J, Delwart E (2014) Feline fecal virome reveals novel and prevalent enteric viruses. Vet Microbiol 171:102-111

18. Niu JT, Yi SS, Hu GX, Guo YB, Zhang S, Dong H, Zhao YL, Wang K (2018) Prevalence and molecular characterization of parvovirus in domestic kittens from Northeast China during 2016-2017. Jpn J Vet Res 66:145-155
19. Paris JK, Wills S, Balzer HJ, Shaw DJ, Gunn-Moore DA (2014) Enteropathogen co-infection in UK cats with diarrhoea. BMC Vet Res 10:13

20. Sabshin SJ, Levy JK, Tupler T, Tucker SJ, Greiner EC, Leutenegger CM (2012) Enteropathogens identified in cats entering a Florida animal shelter with normal feces or diarrhea. J Am Vet Med Assoc 241:331-337

21. Stuetzer B, Hartmann K (2014) Feline parvovirus infection and associated diseases. Vet J 201:150-155

22. Takano T, Takadate Y, Doki T, Hohdatsu T (2016) Genetic characterization of feline bocavirus detected in cats in Japan. Arch Virol 161:2825-2828

23. Yang S, Wang S, Feng H, Zeng L, Xia Z, Zhang R, Zou X, Wang C, Liu Q, Xia X (2010) Isolation and characterization of feline panleukopenia virus from a diarrheic monkey. Vet Microbiol 143:155-159

24. Yi S, Niu J, Wang H, Dong G, Guo Y, Dong H, Wang K, Hu G (2018) Molecular characterization of feline astrovirus in domestic cats from Northeast China. PLoS One 13:e0205441

25. Yi S, Niu J, Wang H, Dong G, Zhao Y, Dong H, Guo Y, Wang $\mathrm{K}, \mathrm{Hu} \mathrm{G}$ (2018) Detection and genetic characterization of feline bocavirus in Northeast China. Virol J 15:125

26. Zeng Z, Liu Z, Wang W, Tang D, Liang H, Liu Z (2014) Establishment and application of a multiplex PCR for rapid and simultaneous detection of six viruses in swine. J Virol Methods 208:102-106

27. Zhang W, Li L, Deng X, Kapusinszky B, Pesavento PA, Delwart E (2014) Faecal virome of cats in an animal shelter. J Gen Virol 95:2553-2564

28. Zheng X, Liu G, Opriessnig T, Wang Z, Yang Z, Jiang Y (2016) Development and validation of a multiplex conventional PCR assay for simultaneous detection and grouping of porcine bocaviruses. J Virol Methods 236:164-169

Publisher's Note Springer Nature remains neutral with regard to jurisdictional claims in published maps and institutional affiliations. 